\title{
A Review on Degradation Modelling and Its Engineering Applications
}

\author{
Ameneh Forouzandeh Shahraki ${ }^{\mathrm{a}}$, Om Parkash Yadav ${ }^{\mathrm{a}, *}$, Haitao Liao ${ }^{\mathrm{b}}$ \\ ${ }^{a}$ Department of Industrial \& Manufacturing Engineering, North Dakota state University, Fargo, ND 58108, USA \\ ${ }^{b}$ Department of Industrial Engineering,University of Arkansas, Fayetteville, AR 72701, USA
}

\begin{abstract}
Degradation modeling is an effective approach for reliability assessment, remaining useful life prediction, maintenance planning, and prognostics health management. Degradation models are usually developed based on degradation data and/or prior understandings of physics behind degradation processes of products or systems. Further, the effects of environmental or operational conditions on degradation processes and the knowledge about the dependency between degradation processes help improve the explanatory capabilities of degradation models. This paper presents a comprehensive review of existing degradation modeling approaches commonly used in engineering applications. To assist practitioners in understanding the concept of degradation modelling, the existing methods are classified into two broad categories: the data-driven approach and physics-based modelling approach. By systematically reviewing these approaches, we highlight their merits, useful applications and limitations. Finally, we provide a summary and indicate several future research challenges in this important area of reliability engineering.
\end{abstract}

Keywords: Degradation modelling; data-driven methods; physics-based approach; multiple degradation processes; random shock;

(Submitted on February 22, 2017; Revised on April 22, 2017; Accepted on April 24, 2017)

(C) 2017 Totem Publisher, Inc. All rights reserved.

\section{Introduction}

Degradation of a system is an inherent process influenced by internal and external factors including environmental and operating conditions. Degradation, such as damage accumulation over time, is usually an irreversible process leading to failure when the accumulated damage exceeds a natural or predetermined threshold level. For a critical system, such failures may cause severe losses. Therefore, it is imperative to understand and model the system's degradation behavior for prediction and prevention of potential failures so that subsequent losses can be effectively avoided. For the past few decades, extensive research has been conducted in the area of degradation modelling for reliability analysis and other applications.

Compared to failure time data, degradation data provide valuable information on product failure behavior for making quick reliability assessment and other logistic decisions. The characteristics of degradation data and the methods used for data collection play a significant role in selecting appropriate degradation models. Generally, such data come from laboratory tests, field applications and/or real-time condition monitoring data collected either at normal operating or at accelerated stress conditions. These data can be the direct measurements of degradation processes (e.g., crack growth, decrease in light intensity of light emitting diodes) or the measurements of other characteristics that are closely related to the product's degradation process (e.g., vibration, change in output voltage, temperature).

Sometimes complex systems may experience multiple degradation processes affected by randomly changing covariates, such as temperature, humidity, and voltage. So far, some research effort has been focused on capturing the interaction and dependency of degradation processes along with their influences on failure propagation, and on the impact of shocks that accelerate these failure processes [Peng et al., 2010; Pan and Balakrishnan, 2011; Liu et al., 2014; Liu et al., 2015; Lin et al., 2016]. In a variety of engineering applications, more and many other complex situations must be dealt with. For a practitioner, it is critical to construct a proper degradation model that can capture the true degradation behavior of a product in the field. 
To this end, a comprehensive understanding about the state-of-the-art is essential.

In recent years, degradation-based reliability analysis has gained momentum for getting some insights into product behavior and reducing the overall product development time. An increasing emphasis on accelerated degradation testing has created a need for further research on degradation modelling. In recent years, efforts have been made to compile and review the current state of research on degradation modelling. Si et al. [2011] focused their review on remaining useful life (RUL) estimation for a product. Their effort confined to statistical data-driven approaches that mostly rely on historical data and statistical models. Ye and Xie [2015] classified existing degradation models into stochastic process models, general path models, and others with a focus on stochastic models. Recently, Zhang et al. [2015] provided a review on degradation model-based RUL estimation approaches for systems with heterogeneity. Because of the increasing interest in degradation-based reliability analysis, it would be valuable to conduct a comprehensive review on the state-of-the-art of degradation modelling.

The main purpose of this review is to categorize the existing literature and summarize the latest research progress to assist researchers and practitioners in acquiring a clear comprehension of the subject area. The existing degradation modelling methods are classified into two broad categories: the data-driven and physics-of-failure based models. Data-driven models are established using statistical fitting to the observed degradation data without considering degradation mechanisms. On the other hand, physics-based models capture the failure mechanisms or physical phenomena to build a mathematical description of the degradation process. This review also covers degradation modelling for complex systems subject to multiple correlated degradation processes, and the recent research on different shock models and their effects on prevailing degradation processes.

The remainder of this paper is organized as follows: section 2 provides a discussion on basic degradation models and analysis methods. The work on modelling multiple degradation processes is summarized in section 3 . Section 4 presents a summary of degradation models considering random shocks. Finally, section 5 concludes this work and provides a brief discussion on future research challenges.

\section{Degradation Models}

The following sub-sections briefly discuss those degradation models in the two categories.

\subsection{Data-driven models}

Data-driven models are becoming increasingly popular when it is difficult to capture and understand the physics behind a degradation process. These models can be classified into two subcategories: statistical models and artificial intelligence models. In the first subcategory, the general path and stochastic process models have been widely used. The artificial intelligence approaches, such as neural networks, have also been used in reliability estimation using degradation data. The following sub-sections provide more detailed discussion and recent advances in each of these subcategories.

\subsubsection{General path model}

In general statistical models for continuous degradation data, also called general path model, the degradation process is described as a function of time, possibly with a set of fixed-effects parameters and a set of random-effects parameters. Lu and Meeker [1993] presented a general nonlinear regression model to characterize the degradation path of a random population of units. The model can be represented as:

$$
y_{i j}=D_{i j}=D\left(t_{i j} ; \varphi, \theta_{i}\right)+\varepsilon_{i j}
$$

where $y_{i j}$ represents the observed degradation, and $D(\cdot)$ represents the actual degradation of the $i^{\text {th }}$ unit at time $\left(t_{i j}\right) . \varphi$ is the vector of fixed-effects parameters (common for all units), $\theta_{i}$ is the vector of random-effects parameters representing the characteristics of the $i^{\text {th }}$ unit, and $\varepsilon_{i j}$ is associated random error of the $i^{\text {th }}$ unit at time $t_{i j}$ which is assumed to be $\varepsilon \sim N\left(0, \sigma^{2}\right)$ with zero mean and variance $\sigma^{2}$.

Several extensions of the general path model have been made by considering different types of statistical modelling approaches for different applications. For example, Freitas et al. [2009] used a linear degradation path model to estimate the lifetime distribution of train wheels. They considered a single random parameter with lognormal, Weibull, and normal 
distributions. Yu [2006] assumed a linear degradation path with a reciprocal Weibull-distributed degradation rate to determine the optimal design of an accelerated degradation test. Although the simplicity of linear random-effects model is an advantage, it might not be a good representative for the actual degradation path compared to a nonlinear random-effects model. Usually, nonlinear models may capture the degradation behavior better, and thus can provide better model fitting to the actual data. Bae and Kvam [2004] modelled the degradation path of highly reliable light display components as a nonlinear randomcoefficient model, which allows for a non-monotonic degradation path to capture the burn-in characteristic of the component. Bae et al. [2007] discussed additive and multiplicative degradation models to derive the lifetime distribution of degraded components. In the additive model, the random effects are added to the mean path of degradation, while in the multiplicative model, the random effects are multiplicative to the mean degradation.

The error term (i.e., $\varepsilon$ ) captures the effect of product and environmental noise, signal transients, measurement errors, and/or variations due to monitoring equipment. It is often assumed to be independent and identically distributed as $N\left(0, \sigma^{2}\right)$. Realizing such an assumption my not be valid for all degradation data, Lu et al. [1997] considered non-constant variance for the error term. Gebraeel et al. [2005] considered both Brownian motion error and independent normal random error models. They showed the Brownian motion error model is more appropriate when the successive errors are correlated.

Realizing that operational and environmental conditions also influence the degradation behavior of a product, Meeker et al. [1998] extended the work of Lu and Meeker [1993] to include covariates into the general path model. There have been several other efforts in this context by modelling the parameters of a basic degradation model as a function of covariates. Pan and Crispin [2011] used a hierarchical Bayes model for investigating the degradation process of light-emitting diodes by varying temperature and forward current in accelerated degradation tests. Gabraeel and Pan [2008] considered additional model parameters to capture the time-varying nature of multiple environmental and operational conditions by assuming that the future environment is deterministic and known. Xu et al. [2015] also proposed a class of general path models by incorporating dynamic covariates. The covariate effects are modelled by shape-restricted splines.

Model parameter estimation is another important issue in developing degradation models. For the general path model, the parameters can be estimated using either the frequentist approach or the Bayesian alternative. A frequentist approach uses historical data only to estimate the model parameters of interest. For example, Lu and Meeker [1993] proposed a two-stage approach to estimate model parameters. In the first stage, a regression method is used to obtain the unknown parameters for each unit. Later, the parameter estimates obtained for each unit are used to estimate the measurement-error variance, fixedeffects parameters, and the random-effects parameters. Later, Wu and Tsai [2000] presented a fuzzy-weighted estimation method to modify the two-stage procedure proposed by Lu and Meeker [1993]. Lu et al. [1997] proposed a model with random regression coefficients and a standard-deviation function for analyzing linear degradation data for semiconductors. They estimated the model parameters using the maximum likelihood estimation (MLE) method. Su et al. [1999] considered a random coefficient degradation model with a random sample size for each product unit and used the MLE for parameter estimation. Unlike the frequentist approach, the Bayesian approach combines the degradation data with a prior belief to derive a posterior distribution of the model parameters. For example, Gebraeel et al. [2005] developed a Bayesian updating method that uses real-time condition monitoring data to update the stochastic parameters of an exponential degradation model. Chen and Tusi [2013] proposed a two-phase model to characterize the degradation process of rotational bearings in an effort to improve the accuracy of degradation modelling and prediction. They used a Bayesian framework to integrate historical data with up-to-date in situ observations on new working units. Recently, Son et al. [2016] proposed a constrained Kalman filtering (CKF) approach to model and update the degradation path. The CKF approach is more effective for modeling degradation signals than the conventional Bayesian model when the degradation signals are significantly contaminated by random noise.

The general path models are widely used due to their simplicity, capability to model continuous processes and allow different variance-covariance structures of the response vector. Nevertheless, sometimes these models might not well describe the actual degradation process because of oversimplification of reality. Further, general path models consider the inherent degradation to be deterministic and thus have difficulty in capturing the time-varying behavior of a product. Their inability to capture the temporal variability and the uncertainty inherent in the progression of deterioration over time limits their engineering applications.

\subsubsection{Wiener processes}

Wiener process is also called Gaussian process or Brownian motion with drift. In general, a Wiener process can be expressed as [Whitmore and Schenkelberg, 1997]: 


$$
W(t)=v \Lambda(t)+\sigma B(\Lambda(t))
$$

where $v$ is the drift parameter showing the rate of degradation, $\sigma$ is the volatility parameter, $B(\cdot)$ is the standard Brownian motion, and $\Lambda(t)$ is a monotone increasing function representing a general time scale. The process $W(t)$ is often used to represent system degradation and has the following properties:

- $W(0)=0$ almost surely;

- $W(t)$ is a continuous process having stationary and independent increments, i.e., $\Delta W(t)=W(t+\Delta t)-W(t)$ is independent of $W(s)$ for $s \leq t$, and $\Delta W(t) \sim N\left(\beta(\Lambda(t+\Delta t)-\Lambda(t)), \sigma^{2}(\Lambda(t+\Delta t)-\Lambda(t))\right)$.

Due to its useful mathematical properties and physical interpretations, the Wiener process has been extensively used for modeling degradation processes [Wang, 2010; Whitmore, 1995]. When the mean of degradation is linearly increasing, the Wiener process with linear drift is used. Further, the distribution of the first passage time, i.e., the time when the degradation process first reaches the critical failure threshold, follows an inverse Gaussian distribution. For some nonlinear degradation processes, the mean degradation path can be linearized using appropriate transformation. For example, time-scale transformation was applied to model the degradation of transistors [Whitmore, 1995] and beam bridges [Wang, 2010]. In Elwany and Gebraeel [2009], log-transformation was applied to the nonlinear degradation data. However, not all nonlinear degradation processes can be properly linearized. Recently, nonlinear structures have been proposed to capture the dynamics of nonlinear degradation processes [Si et al., 2012; Wang et al., 2014]. Furthermore, Wang et al. [2014] proposed a general degradation-modelling framework for hybrid deteriorating systems, which have both linear and nonlinear degradation components.

The temporal variability, measurement variability, and unit-to-unit variability are three sources of variation that affect degradation modelling [Zheng et al, 2016]. Wiener process can capture inherent uncertainty associated with the progression of degradation over time. To consider the effects of measurement errors, Whitmore [1995] added an error term to the basic model Eq. (2) by assuming measurement errors are independent and identically distributed following a normal distribution. Later, Ye et al. [2013] showed that the approach in Whitmore [1995] does not make full use of the data and proposed a modified Wiener process with mixed effects. Recently, researchers have proposed random-effects Wiener process models to capture the heterogeneity or unit-to-unit variability within the product population. Peng and Tseng [2009] incorporated the random effects of a drift coefficient and measurement errors into a Wiener process. They assumed that different units have different drift parameter values of $v$ while having the same volatility parameter $\sigma$. Furthermore, to reduce the computational effort, Si et al. [2013] assumed that the drift parameter $v$ is normally distributed. Peng and Tseng [2013] used a skewed-normal distribution to model drift coefficients. All the models discussed so far assume volatility parameter $\sigma$ as a constant for all units. However, in many real-world applications, the correlation between drift and volatility parameters cannot be ignored. Wang [2010] used a Gamma distribution to model the volatility parameter of a Wiener process. Ye et al. [2015] introduced a new class of Wiener process models to capture the correlation between $v$ and $\sigma$. They added a common parameter in the mean and variance of Wiener process to model the correlation. More recently, Zheng et al. [2016] presented a general nonlinear degradation model to characterize the degradation nonlinearity and to capture the variability attributed to all three sources simultaneously.

Additionally, efforts have been made to include the influence of environmental and other operating stress factors in Weiner process models. Initially, it was assumed that drift parameter $v$ is a function of these covariates and volatility parameter $\sigma$ is assumed to be a constant [Liao and Tian, 2013; Liu et al., 2017]. For example, Liao and Tian [2013] considered a single degrading unit under time-varying operating conditions. They modelled the degradation process as a Brownian motion with instantaneous drift parameter governed by an acceleration function, such as a linear model, Arrhenius model, or inverse power law [Elsayed, 1996]. Bian et al. [2015] proposed an approach to take into account environment/operating conditions that are stochastically evolving according to a continuous-time Markov chain (CTMC). For any given unit, the degradation rate is a function of the state of CTMC. Later on, Ye et al. [2015] considered cases where both the drift and volatility parameters are dependent on changing environmental conditions according to respective stress-acceleration relationships.

Although Wiener processes have been used to model many degradation phenomena, they are not suitable for modelling monotonic degradation processes, such as wear or cumulative damage processes. In addition, a one-phase Wiener process is not applicable for many devices exhibiting multi-phase degradation. In fact, the degradation rates of such devices may change at some point in time due to the change in degradation mechanism. To overcome the limitation of Wiener process model, Bae et al. [2015] proposed two-phase and multi-phase Wiener processes to model distinct phases of degradation. 


\subsubsection{Gamma processes}

Abdel-Hameed [1975] first suggested Gamma process as a useful model for degradation processes. It is appropriate to use Gamma process when the gradual damage is monotonically increasing or decreasing over time, such as fatigue, corrosion, and crack growth [Grall et al., 2002; Lawlee and Crowder, 2004]. The basic Gamma process $\{\mathrm{Y}(\mathrm{t})$; $\mathrm{t} \geq 0\}$ with shape function $\eta(t)>0$ and scale parameter $\mu>0$ is a continuous-time stochastic process with the following properties:

- $\mathrm{Y}(0)=0$ with certainty;

- $\quad \mathrm{Y}(\mathrm{t})$ has independent non-negative increments, i.e., $\mathrm{Y}(\mathrm{t}+\mathrm{u})-\mathrm{Y}(\mathrm{u})$ and $\mathrm{Y}(\mathrm{s}+\mathrm{v})-\mathrm{Y}(\mathrm{v})$ are independent for $\mathrm{t}+\mathrm{u}>\mathrm{u} \geq$ $\mathrm{s}+\mathrm{v}>\mathrm{v}$;

- $\quad \mathrm{Y}(\mathrm{t}+\mathrm{u})-\mathrm{Y}(\mathrm{u}) \sim \operatorname{Gamma}(\eta(\mathrm{t}+\mathrm{u})-\eta(\mathrm{u}), \mu)$, where $\eta(\mathrm{t})$ is a monotone increasing function with $\eta(0)=0$.

Van Noortwijk [2009] reviewed the application of Gamma process in maintenance, its statistical properties, and parameter estimation methods. Gamma process-based models can easily manage temporal variability in degradation process [Pandey et al., 2009], while the unit-to-unit variability can be modelled by introducing random effects into the basic model. For example, Lawless and Crowder [2004] proposed Gamma process-based models by assuming that the scale parameter is a random variable that follows a Gamma distribution. They used the proposed model to take into account different degradation rates of individual units. Ye et al [2014] considered the unit-to-unit variability and used the score statistic to test the existence of random effects. Moreover, Lu et al. [2013] presented an efficient estimation method to filter out the effect of random noise from the measured degradation data. Recently, Le Son et al. [2016] modelled system deterioration by a non-homogeneous Gamma process and used Gibbs sampling to approximate the hidden degradation states contaminated by noise. Pulcini [2016] proposed a perturbed Gamma process by assuming measurement errors are normally distributed with mean zero and standard deviation that depends on the actual degradation level.

Further, Bagdonavicius and Nikulin [2001] introduced the effects of environmental stress factors into Gamma process models by defining the covariate-dependent shape function. Lawless and Crowder [2004] considered the scale parameter of Gamma process as a function of covariates. Van Noortwijk [2009] also considered a parametric form for the shape function. Recently, Peng et al. [2015] modelled the effects of different usage conditions and time-varying missions coherently. They incorporated accelerating variables into the shape function and modified the shape function by incorporating the effect of mission intensity. As a result, the shape function depends on both the operation time and the mission type.

Regarding parameter estimation, the conventional methods such as MLE, method of moments, and Bayesian method can be used to estimate the parameters of a Gamma process. Guo and Tan [2009] proposed a Bayesian approach to update parameter estimates when additional information becomes available. Ye et al. [2014] obtained the MLE through an expectation maximization (EM) algorithm. They also showed that estimating the shape function $\eta(\cdot)$ in a nonparametric manner does not require prior knowledge about $\eta(\cdot)$.

The use of Gamma process for degradation modelling is getting popular as it has a physical interpretation and its mathematical representations are straightforward. Moreover, the model takes into account the temporal variability of a degradation process. On the other hand, its Markov property and the fact that it is strictly applicable to monotonic processes may restrict its application for some degradation processes.

\subsubsection{Inverse Gaussian processes}

The inverse Gaussian (IG) process has been used to model monotone degradation data when other processes do not fit the data very well such as GaAs laser degradation data [Wang and $\mathrm{Xu}, 2010$ ] and energy pipeline corrosion data [Qin et al., 2013]. The basic IG process $\{\mathrm{Y}(\mathrm{t}) ; \mathrm{t} \geqslant 0\}$ has the following properties:

- $\mathrm{Y}(0)=0$ with certainty;

- $\mathrm{Y}(\mathrm{t})$ has independent increments, i.e., $\mathrm{Y}\left(\mathrm{t}_{2}\right)-\mathrm{Y}\left(\mathrm{t}_{1}\right)$ is independent of $\mathrm{Y}\left(\mathrm{t}_{4}\right)-\mathrm{Y}\left(\mathrm{t}_{3}\right)$ for $\mathrm{t}_{4}>\mathrm{t}_{3} \geq \mathrm{t}_{2}>\mathrm{t}_{1} \geq 0$.

- Each increment follows an IG distribution, that is, $Y(t)-Y(s) \sim I G\left(\mu(\Lambda(\mathrm{t})-\Lambda(\mathrm{s})), \lambda(\Lambda(\mathrm{t})-\Lambda(\mathrm{s}))^{2}\right)$ for $\mathrm{t}>\mathrm{s} \geqslant 0$, where $\Lambda(\mathrm{t})$ is nonnegative and monotone increasing function of time $(\Lambda(0)=0)$.

The $I G$ probability density function $\operatorname{IG}\left(\mu \Lambda(\mathrm{t}), \lambda \Lambda(\mathrm{t})^{2}\right)$ is defined as:

$$
f_{I G}(y ; \mu, \lambda)=\sqrt{\frac{\lambda \Lambda(t)^{2}}{2 \Pi y^{3}}} \exp \left\{\frac{-\lambda}{2 y}\left(\frac{y}{\mu}-\Lambda(t)\right)^{2}\right\} y, \lambda>0, \mu \in R
$$


where the process parameters, $\mu$ and $\lambda$, represent the degradation rate and degradation volatility, respectively.

Recently, IG process has received more attention in modelling degradation data because of its mathematical properties and flexibility in dealing with random effects and covariates. Wang and Xu [2010] proposed IG process to model the degradation data of GaAs lasers by incorporating both the unit-to-unit variability and covariate information into the model. Later, Ye and Chen [2014] developed two other random-effects models to make IG process more flexible than Gamma process in considering unit-to-unit variability. They also attempted to explain the physical meaning of IG process by presenting the relationship between the IG process and the compound Poisson process. Further, Peng et al. [2014] improved the randomeffects models by modifying the parameter structure of the simple IG process model to consider the situations where the random effects influence only the mean value of the degradation process. Recently, Peng [2015] also proposed an inverse normal-gamma mixture of an IG process model.

Regarding model parameter estimation, Wang and Xu [2010], Ye and Chen [2014], and Peng [2015] used the MLE method and EM algorithm and bootstrap approach. Peng et al. [2014] constructed a general Bayesian framework for degradation analysis with IG process models.

Interestingly, there is an inverse relationship between Wiener and IG processes that allows many of Wiener process properties to be extended to the IG process. Moreover, the IG process is flexible for incorporating random effects and covariates in degradation data analysis. It is easier to determine the probability density function and cumulative distribution function of RUL analytically in an IG process model.

\subsubsection{Finite-state degradation models}

Unlike the Wiener process, Gamma process and inverse Gaussian process models, a finite-state Markov process is a stochastic process that evolves through a finite number of states. Due to the Markov property, the future state of the process is independent of past states given the current state. In Markovian-based degradation models, the transition probabilities (or rates) depend only on the states involved in the transition. In real-world applications, however, the transition probabilities (or rates) may also depend on other factors, such as the actual level of degradation, the time when the product reached the current state, the sojourn time, the total age of the system, and some covariates [Chen and $\mathrm{Wu}, 2007$; Chen, 2011]. Semi-Markovian models extend the application of Markovian-based models by incorporating the effects of these factors. Moghaddass and Zuo [2012] and Moghaddass et al. [2012] classified semi-Markov processes into four categories based on the type of transitions as shown in Figure 1.

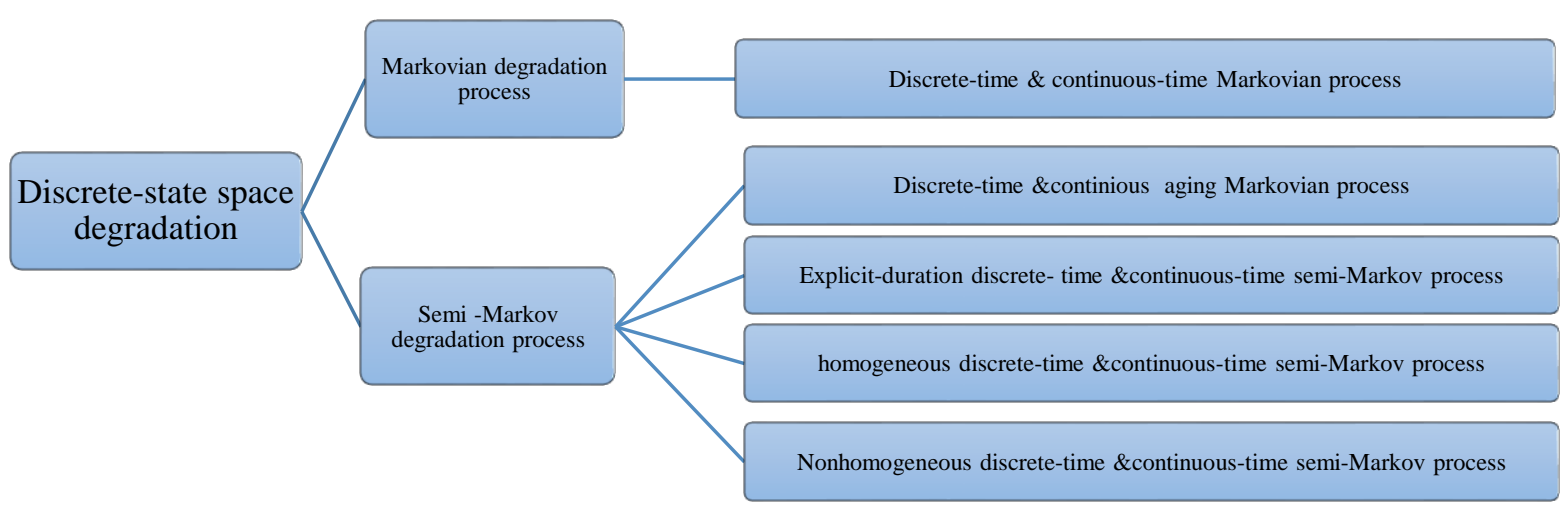

Figure 1. Different categories of discrete-state space degradation models

Discrete-time and continuous-time semi-Markov processes represent two classes of semi-Markov processes [Chryssaphinou et al., 2011; Compare et al., 2016]. In homogeneous discrete-time and continuous-time semi-Markov processes, the time spent at the current state can affect the time-dependent transition rate (or the transition probability for a 
discrete-time process) between states [Kharoufeh et al., 2010]. In these models, however, the transition rates (or the transition probability for a discrete-time process) are independent of the age of the system. Therefore, these models are not applicable when the degradation process depends on both the sojourn time at each state and the total age of a system. On the other hand, nonhomogeneous discrete-time and continuous-time semi-Markov processes provide more general models that cover many other structures [Moghaddass et al., 2012]. For example, the degradation transitions between states in a single system can follow non-identical structures and the transitions between states can depend on any combination of influential factors.

All the models depicted in Figure1 are based on the assumption that the degradation process is directly observable. However, in many cases, the degradation level is not directly accessible due to the complexity of degradation process or nature of the product type. To deal with such cases, hidden Markov models (HMM) and hidden semi-Markov models (HSMM) have been developed.

The HMM deals with two different stochastic processes: an unobservable degradation process and a measurable characteristic that is dependent on the actual degradation process. In HHMs, finding a stochastic relationship between the degradation process and the observation process is essential for condition monitoring and reliability analysis. HMMs have been used successfully in a wide range of applications. For example, Bunks and McCarthy [2000] first discussed using HMMs for condition-based maintenance. Baruah and Chinnam [2005] applied HMMs to carry out both diagnostic and prognostic for metal cutting tools. They identified the health state of the cutting tool by modelling vibration signals as the observation process. Wang [2007] presented a wear prediction model based on stochastic filtering and hidden Markov theory to describe the relationship between the wear process and the observed oil condition using a floating scale parameter. Despite a rich mathematical structure of HMMs and many successful applications, the Markov property and constant transition rates do not allow HMMs to model temporal structures.

Some of the limitations of HMMs were managed in HSMMs by allowing the underlying unobserved state process to be a semi-Markov chain with a variable duration or sojourn time for each state. This consideration provides more flexibility for incorporating and modelling temporal structures [Yu, 2010]. Dong et al. [2006] developed an integrated methodology based on HSMMs for equipment health diagnosis and prognosis. Furthermore, Dong and He [2007] presented an approach for performing multi-sensor equipment diagnosis and prognosis by modelling the duration of a health state using an explicit Gaussian probability function. They also introduced new forward and backward variables to improve computational efficiency. However, these methods assume that the state transition probabilities would remain constant without considering the age of the product. To address this issue, Peng and Dong [2011] proposed an age-dependent HSMM-based prognosis method to predict equipment health. In another extension, a nonhomogeneous continuous-time HSMM process was used for modelling the degradation and observation processes [Moghaddass et al., 2012]. These models essentially consider that the transition between two states can depend on the two states involved in the transition, the time that the last state was reached, the time spent at the current state, the total age of the system, and some covariates involved.

A few other researchers have also considered time-varying environmental or operating conditions and updated the model parameters using on-line data. Zhou et al. [2010] proposed a new prognosis model by combining the capabilities of the HMM and belief rule base (BRB) to model the effects of environmental factors on degradation states. They used the BRB to establish the relationships between the environmental factors and the transition probabilities among the hidden states of the system. Cholette and Djurdjanovic [2014] presented a context-dependent degradation modelling based on the concept of HMM. Their methodology utilizes historical information about known operational conditions to train a series of operation-specific HMMs. Recently, Zhang et al. [2016] presented a condition-based monitoring methodology based on operating regime-specific HMMs of system degradation. The parameters of HMM are identified along with the corresponding estimation uncertainty using a Bayesian estimation approach. Regarding HSMMs, Liu et al. [2012] proposed a new multi-step-ahead health recognition algorithm based on a joint probability distribution. They enhanced the capability of HSMM for online health prognostic of equipment using a sequential Monte Carlo method. Later, Liu et al. [2015] proposed an adaptive HSMM for health prognostic of multi-sensor monitoring equipment to reduce the computation and space complexity.

Despite the extensive use of Markovian-based models for degradation modelling, a few limitations restrict their wider applications. From a practical application point of view, defining the discrete degrading states and estimating corresponding transition probabilities or rates has been one of the biggest challenges.

\subsubsection{Other models}

Artificial intelligence methods, such as neural networks and fuzzy logic, belong to another set of data-driven models for degradation modelling and prediction. A neural network model learns from given inputs in a way to produce the desired 
outcome. After learning from historical data, the network model can be used to predict system status. Gebraeel and Lawley [2008] developed a neural network-based degradation model for computing the remaining life distributions of partially degraded components and updated those using in-situ condition monitoring signals. Malhi et al. [2011] applied recurrent neural networks for long-term prediction of defect progression in machines. For the first time, Fink et al. [2014] applied multilayer feed-forward neural networks with multi-valued neurons to degradation modelling and reliability prediction. The main benefit of using artificial neural networks is its ability to model complex multidimensional degradation signals, for which analytic models are difficult to obtain. However, the requirement for large amounts of training data limits the application of neural networks. Kan et al. [2015] reviewed the application of artificial neural networks and discussed thoroughly their advantages and limitations.

Another set of data-driven models uses fuzzy logic that maps an input data vector to a scalar output to handle non-linear, non-stationary, and complex system modelling. Zio and Di Maio [2010] proposed fuzzy similarity analysis for estimating the RUL of a system. Neuro-fuzzy systems, the combination of fuzzy logic and neural networks, have been used in many studies to take the advantage of both fuzzy logic and neural networks [Zhao et al., 2009; Soualhi et al., 2014].

Filtering-based models provide another approach to modelling unobservable degradation processes using observed condition monitoring (CM) data or signals. In particular, Kalman filter considers the unobserved condition, $x_{t}$, and the observed CM data, $y_{t}$, following the state space equations: $x_{t}=\alpha x_{t-1}+\epsilon_{t}$ and $y_{t}=\beta x_{t}+\eta_{t}$, where $\epsilon_{t}$ and $\eta_{t}$ are Gaussian noises, and $\alpha$ and $\beta$ are the model parameters [Jouin et al., 2016]. Extended Kalman filter, an extension to Kalman filter, can be applied for nonlinear systems [Kan et al., 2015]. In addition, particle filter, also referred to as sequential Monte Carlo, can be used for a nonlinear system with non-Gaussian noise [Jouin et al., 2016].

\subsection{Physics-based approach}

Physics-based models consider a product's failure mechanisms in describing the degradation process. These models quantitatively characterize the product's failure behavior using physics laws. The application of physics-of-failure (PoF) models require clear understandings of failure mechanisms and potential failure locations at a particular loading condition. The loading condition could be thermal, mechanical, chemical, electrical, and other types of stresses responsible for the failure process. For electronic devices, typical failure mechanisms are corrosion, fatigue, electromigration, conductive filament formation, stress-driven diffusion voiding, and time-dependent dielectric breakdown. The primary failure mechanisms in mechanical components include material yield, stress fracture, corrosion, wear, creep, shock and fatigue.

A majority of physics-based degradation models consider available PoF models, such as Arrhenius, Eyring, and Inverse Power models [Meeker et al., 1998; Fan et al., 2011]. Arrhenius model has been widely used when the acceleration variable is temperature. Eyring model is often used for incorporating temperature and one or more additional stress variables. Inverse power model has been widely used to estimate the RUL of electronic devices when the accelerating variables are non-thermal. In addition, the Paris crack growth [Paris and Erdogan, 1963] and Forman crack growth models [Forman et al., 1967] are the two commonly used models for structural elements that establish the relationship between the crack growth rate and stress.

Several efforts have been made in the past to model degradation phenomena using PoF model-based approaches. Pecht and Dasgupta [1995] presented an overview of PoF models for electronic devices and discussed a case study of a conductive filament formation failure mechanism in printed circuit boards. Adams [2002] modelled the damage accumulation in a dynamic structural system using first and second order nonlinear differential equations. Gu et al. [2007] proposed a PoF-based prognostic methodology to estimate the RUL of printed circuit boards subjected to cyclic/vibration loads. Kulkarni et al. [2013] developed a physics-based degradation model for electrolytic capacitors under thermal stress conditions. Yang et al. [2013] reviewed the use of PoF methods on wire bonding interconnects in power electronic modules and addressed the effect of exposure duration to the temperature loads on the bond degradation rate. Further, they developed a model for wire bond fatigue, which could analyze the cycle-to-failure under different steady-state temperature and thermal cycle conditions.

The PoF models were also employed to model the degradation processes of mechanical components, such as rolling element bearing, rotor shaft, and ball bearings. Li et al. [2014] used the Paris-Erdogan model to characterize the dynamic evolution of a rolling element bearing. Oppenheimer and Loparo [2002] modelled a rotor shaft crack growth using the Forman law of linear elastic fracture mechanics and used the model for predicting its health condition. A stress-based fatigue life model is proposed by Yu and Harris [2001] to model the life of ball bearings. Recently, Zhao et al. [2013] developed an integrated gear prognosis method by combining both physics-based models and real-time condition monitoring data. They modelled the crack propagation using the Paris' law, and an error term is added to model uncertainty. Further, the PoF models were combined with probabilistic techniques to address the limitations of data availability and to capture randomness or 
uncertainties. To capture loading patterns and conditions, Rathod et al. [2011] proposed a methodology for modelling the probabilistic distribution of the damage accumulation measure and failure time to assess product reliability. Guang et al. [2012] proposed a stochastic PoF method to determine the momentum of a wheel subject to accelerated testing.

Several methods have been proposed to estimate model parameters, including Kalman filter, extended Kalman filter, and particle filter methods [Swanson, 2000; Zio and Peloni, 2011]. These three methods are based on the filtering technique, which update model parameters recursively by taking one measurement data at a time. An et al., 2011 presented a statistical model parameter identification approach using Bayesian inference when the model parameters are correlated and the observed data are biased and contaminated by noise.

Generally, the physics-based methods are quite useful due to their ability to capture a physical phenomenon. Compared to data-driven approaches, this approach provides a better means to handle the bias in measured data and is able to explain the behaviour of a system in a wide range of operating condition. On the other hand, the PoF models require a complete understanding of the failure mechanisms and theories. Therefore, it is difficult to develop such models for the damage evolution processes of complex systems.

\section{Modeling Multiple Degradation Processes}

Failures of a complex engineering system may be caused by multiple degradation processes. As a result, it is important to consider multiple degradation processes simultaneously for system reliability assessment. Some of the earlier work on multiple degradation modelling assumed that the degradation measures are either independent [Crk, 2000] or dependent with a multivariate normal distribution [Wang and Coit, 2004]. For example, Crk [2000] assumed that system failure is governed by several independent mechanisms and presented an effective way to estimate the system's reliability by monitoring each degradation measure. In practice, assuming independent degradation processes eases the modeling of multiple degradation processes, but it may not be realistic and can lead to poor or ineffective modelling of component and system reliability. To address this concern, Wang and Coit [2004] proposed a multivariate degradation model based on a multivariate normal distribution. Recently, the use of copula in modelling dependent degradation measures has gained more attention. Pan and Balakrishnan [2011] adopted a bivariate Birnbaum-Saunders distribution and its marginal distributions to approximate the reliability function of a system with two Gamma degradation processes. Sari [2007] showed the use of copula to model the dependency between two or more degradation processes and modelled the degradation data with a generalized linear model.

Recently, Pan et al. [2013] introduced bivariate degradation models based on Wiener processes and captured the dependency among stochastic degradation models via a copula. The parameters of the two degradation models and the copula were estimated jointly using Bayesian Markov Chain Monte Carlo method (Bayesian MCMC). Liu et al. [2014] employed the copula method to fit the joint distribution of multiple degradation processes, and used an EM algorithm to estimate model parameters. Furthermore, Peng et al. [2016a] introduced a new type of bivariate degradation process model based on the IG process and copula. They presented a two-stage Bayesian method to estimate the model parameters for both complete and incomplete degradation data. Subsequently, Peng et al. [2016b] proposed a generalized multivariate hybrid degradation process model that considers both monotonic and non-monotonic degradation processes and captures the influence of environmental and loading profiles.

Some researchers have attempted to model degradation processes of systems comprising of stochastically dependent components. The limited literature that considers stochastic dependency can be divided into two groups. In the first group, an external event or the failure of one component triggers the stochastic dependency between components. The triggering event can influence those affected components in three possible ways: leads to an immediate failure with a certain probability, increases degradation rate, or changes the degradation levels of remaining affected components [Nicolai and Dekker, 2008]. In $\mathrm{Li}$ et al. [2011], the stochastic dependency among multiple degradation processes was modelled by defining each component's degradation path as a combination of an independent degradation mean and common random degradation factors. They limited the dependency between components to the shared environmental or operating stresses. Recently, Liang and Parlikad [2015] considered a multi-component system consisting of critical and non-critical components. They modelled both normal degradation and accelerated degradation processes of the system using a CTMC with the understanding that the malfunction of a noncritical component leads to the transition of a critical component from its normal degradation path to the accelerated degradation path.

Another group of models focuses on scenarios where the degradation behaviour of components is influenced by one another. To model this phenomenon, Bian and Gebraeel [2014a] considered that the degradation level of one component affects the degradation rate of other components in the system. They assumed that when the degradation signal of one component reaches a certain value, it triggers a change in the degradation rate of other interdependent components. Later, 
Bian and Gebraeel [2014b] extended their previous work by considering the fact that component interactions can occur on a continuous basis. They modelled multivariate component degradation signals using a linear system of stochastic differential equations. Similarly, Eryilmaz [2014] considered two-component systems where degradation processes of the two multi-state components follow a Markov process, and modelled the dependence of the components using a copula. Recently, Dao and Zuo [2016] considered a multistate series system with two types of stochastic dependency among components: the failure of a component can instantly cause complete failure of other components, and a component's degradation affects the state and the degradation rate of components.

\section{Degradation Models Considering Random Shocks}

Random shocks represent an exogenous process that may affect system degradation. Usually, random shocks are independent but can propagate failure process by influencing the degradation rate of a degradation process and in some cases, initiating failure processes. Initially, researchers attempted to model both degradation processes and random shocks as independent competing failure processes. For example, Klutke and Yang [2002] proposed an availability model for a system exposed to both degradation and shocks. In addition, other researchers analyzed systems by assuming independent failure processes including both degradation processes and random shocks [Li and Pham, 2005].

To consider the interaction between random shocks and degradation processes, Peng et al. [2010] considered two dependent failure processes: soft failures caused jointly by continuous degradation and a sudden increase in degradation level caused by a shock, and catastrophic failures caused by an abrupt and excessive stress from the same shock process. Wang and Pham [2011] proposed a dependent competing risk model for a deteriorating system subject to shocks. They assumed that shocks follows a nonhomogeneous Poisson process with an increasing rate of occurrence based on the fact that an aged system is more vulnerable to the shocks. Similarly, Wang et al. [2011] considered the influence of a periodic shock process on the degradation process. The effect of shock on performance causes either a sudden increase in the failure rate or a change in the degradation level after the occurrence of a shock.

Later, Jiang et al. [2012] extended the work of Peng et al. [2010] by considering two kinds of dependency between soft and hard failure processes: 1) both processes are dependent due to the same shock process, and 2) the hard failure threshold level is dependent on the shock process. Liu et al. [2013] considered both soft and hard failures assuming that the failure rate of hard failure depends on the age and/or the system's degradation state. Rafiee et al. [2014] modelled the effect of shocks on the degradation rate considering different shock models: extreme shock model, $\delta$-shock model, m-shock model, and run shock model. Recently, Jiang et al. [2015] investigated two dependent competing failure processes and categorized shocks into different shock zones. Their model is more realistic as shock loads outside the range of system resistance tolerance are assumed to cause abrupt damage.

For more complex systems, they may experience multiple degradation processes and shocks, making reliability modelling more difficult. Wang and Pham [2012] developed an s-dependent competing risk model for a system subject to multiple degradation processes and random shocks using time-varying copula. They used a time-scaled covariate factor to adjust the correlation between degradation processes and random shocks. Similarly, Song et al. [2014] developed a new multicomponent system reliability model subject to multiple dependent competing failure processes. Lin et al. [2016] considered a system having both continuous and multi-state degradation processes subject to random shocks. The dependency among degradation processes and the influence of random shocks on these processes was addressed by piecewise-deterministic Markov process modelling.

Most of the earlier work on degradation models considering random shocks assumed linear degradation processes. Recently, Jiang et al. [2015] and Caballe et al. [2015] used Gamma processes to model nonlinear degradation. In addition, Kim et al. [2013] and Lin et al. [2016] attempted to model nonlinear degradation using a Markov process. The random shocks have been modelled using either a homogeneous [Peng et al., 2010; Wang and Pham, 2012] or a non-homogeneous Poisson process [Caballe et al., 2015]. Furthermore, Bian and Gerbaeel [2015] considered the possibility that shocks may occur at environment transition epochs (instead of randomly) causing upward or downward changes in the level of degradation.

In summary, significant efforts have been taken on modelling the effects of random shocks on degradation processes. However, most of the work does not consider the dependency between a sudden change in degradation rate or level caused by random shocks and the current degradation level. After all, it is often true that weaker systems with a higher level of degradation are more vulnerable to shocks and/or sudden excessive loads. 


\section{Conclusion and Further Research}

This paper presents a comprehensive review of degradation modelling methods. In particular, the current literature is classified into two broad categories: data-driven and physics-based degradation models. Different models for a single degradation process and the related work are provided for each of these categories. In addition, this review also includes the methods for modelling multiple degradation processes and the effect of random shocks on degradation processes

The modelling techniques developed so far need to be improved further to deal with more complex and hybrid systems. It is believed that there is a need to address the following future research concerns.

- Most of degradation models are developed based on several assumptions that simplify model formulation and reduce the computational complexity. In particular, most of the current literature deals with a single degradation process. However, new hybrid technologies consisting of mechanical, electronic, software and several other elements result in more complex physical systems. These elements, sometimes miniaturized elements, of complex physical systems exhibit multiple degradation processes. To deal with multiple degradation processes, which could be dependent, in a complex system, more advanced stochastic models and statistical methods need to be developed with the help of the increasing capability of modern computational technology.

- Engineering systems usually operate in time-varying operating conditions. The variation in operating conditions causes degradation processes to evolve in a complex manner. It is, therefore, important to develop loading-dependent degradation models that are able to capture the degradation behaviour of the systems in response to the field operating conditions.

- Both data-driven and physics-based models have some unique advantages over each other. Degradation modelling capabilities can be further improved by combining the two types of models. A hybrid degradation modelling approach essentially takes the advantages of both modelling approaches. Some effort has been put in this direction, but more research needs to be done to assist practitioners in utilizing their knowledge about PoF while enhancing data analysis using advanced statistical methods.

- The advancement in sensor technology has made it possible to simultaneously collect real-time data on multiple degradation processes. Such data could be collected from regions with different operating environments. The huge amount of data may be in different forms, such as time series or images. Those multi-dimensional data provide an excellent opportunity for future research on building more realistic degradation models for reliability prediction. This will require more refined analytical techniques to handle multi-dimensional data, and advanced data fusion techniques, such as multi-dimensional covariate analysis, noise reduction and Bayesian analysis.

- New emerging research issues that need to be addressed are related to decision making in logistics. The analysis of real-time degradation data of a system in the field can facilitate RUL prediction, real-time maintenance planning, and effective spare parts inventory management. To this end, more research is required to integrate degradation modelling into logistic decision-making strategies.

\section{References}

1. Z. Pan, and N. Balakrishnan, "Reliability Modeling of Degradation of Products with Multiple Performance Characteristics Based on Gamma Processes," Reliability Engineering \& System Safety, vol. 96, no. 8, pp. 949-957, 2011

2. Z. Liu, X. Ma, J. Yang, and Y. Zhao, "Reliability Modeling for Systems with Multiple Degradation Processes using inverse Gaussian process and copulas," Mathematical Problems in Engineering, 2014

3. H. Peng, Q. Feng, and D.W. Coit, "Reliability and Maintenance Modeling for Systems Subject to Multiple Dependent Competing Failure Processes," IIE transactions, vol. 43, no. 1, pp. 12-22, 2010

4. Y.H. Lin, Y.F. Li, and E. Zio, "Reliability Assessment of Systems Subject to Dependent Degradation Processes and Random Shocks," IIE transactions, vol. 48, no. 11, pp. 1072-85, 2016

5. K. Liu, A. Chehade, and C. Song, "Optimize the signal quality of the composite health index via data fusion for degradation modeling and prognostic analysis," IEEE Transactions on Automation Science and Engineering, vol. 99, no. 1, pp. 1-11, 2015

6. X.S. Si, W. Wang, C.H. Hu, and D.H. Zhou," Remaining Useful Life Estimation-A Review on The Statistical Data Driven Approaches.," European journal of operational research, vol. 213, no. 1, pp. 1-14, 2011

7. Z.S. Ye, and M. Xie, "Stochastic Modelling and Analysis of Degradation for Highly Reliable Products," Applied Stochastic Models in Business and Industry, vol. 31, no. 1, pp. 16-32, 2015

8. Z. Zhang, X. Si, C. Hu, and X. Kong, "Degradation modeling-based remaining useful life estimation: A review on approaches for systems with heterogeneity," Proceedings of the Institution of Mechanical Engineers, Part O: Journal of Risk and Reliability, vol. 229 , no. 4 , pp. 343-355, 2015

9. C.J. Lu, and W.O. Meeker, "Using Degradation Measures to Estimate a Time-To-Failure Distribution," Technometrics, vol. 35, 
no. 2, pp. 161-174, 1993

10. M.A. Freitas, M.L. de Toledo, E.A. Colosimo, and M.C Pires, "Using Degradation Data to Assess Reliability: A Case Study On Train Wheel Degradation," Quality and Reliability Engineering International, vol. 25, no. 5, pp. 607-629, 2009

11. H.F. Yu, "Designing an Accelerated Degradation Experiment with A Reciprocal Weibull Degradation Rate," Journal of statistical planning and inference, vol. 136, no. 1, pp. 282-297, 2006

12. S.J. Bae, and P.H. Kvam, “A Nonlinear Random-Coefficients Model for Degradation Testing,” Technometrics, vol. 46, no. 4, pp. 460-469, 2004

13. S.J. Bae, W. Kuo, and P.H. Kvam, "Degradation Models and Implied Lifetime Distributions," Reliability Engineering \& System Safety, vol. 92, no. 5, pp. 601-608, 2007

14. J.C. Lu, J. Park, and Q. Yang, "Statistical Inference of a Time-To-Failure Distribution Derived from Linear Degradation Data," Technometrics, vol. 39, no. 4, pp. 391-400, 1997

15. N.Z. Gebraeel, M.A. Lawley, R. Li, and J.K. Ryan, "Residual-Life Distributions from Component Degradation Signals: A Bayesian Approach,” IIE Transactions, vol. 37, no. 6, pp. 543-557, 2005

16. W.Q. Meeker, L.A. Escobar, and C.J. Lu, “Accelerated Degradation Tests: Modeling and Analysis," Technometrics, vol. 40, no. 2, pp. 89-99, 1998

17. R. Pan, and T. A. Crispin, "A Hierarchical Modeling Approach to Accelerated Degradation Testing Data Analysis: A Case Study," Quality and Reliability Engineering International, vol. 27, no. 2, pp. 229-37, 2011

18. N. Gebraeel, and J. Pan, "Prognostic Degradation Models for Computing and Updating Residual Life Distributions in A TimeVarying Environment.," IEEE Transactions on Reliability, vol. 57, no. 4, pp. 539-550, 2008

19. Z. Xu, Y. Hong, and R. Jin, "Nonlinear General Path Models for Degradation Data with Dynamic Covariates," Applied Stochastic Models in Business and Industry, 2015

20. S.J. Wu, and T.R. Tsai, "Estimation of Time-To-Failure Distribution Derived from A Degradation Model Using Fuzzy Clustering," Quality and Reliability Engineering International, vol. 16, no. 4, pp. 261-270, 2000

21. C. Su, J.C Lu, D. Chen, and J. M. Hughes-Oliver, "A Random Coefficient Degradation Model with Random Sample Size," Lifetime Data Analysis, vol. 5, no. 2, pp. 173-183, 1999

22. N. Chen., and K. L. Tsui," Condition Monitoring and Remaining Useful Life Prediction Using Degradation Signals: Revisited," IIE Transactions, vol. 45, no. 9, pp. 939-52, 2013

23. J. Son, S. Zhou, C. Sankavaram, X. Du., and Y. Zhang, "Remaining Useful Life Prediction Based On Noisy Condition Monitoring Signals Using Constrained Kalman Filter," Reliability Engineering \& System Safety, vol. 152, pp. 152-38, 2016

24. G.A. Whitmore, and F. Schenkelberg, "Modelling Accelerated Degradation Data Using Wiener Diffusion with A Time Scale Transformation.," Lifetime data analysis, vol. 3, no. 1, pp. 27-45, 1997

25. X. Wang, "Wiener Processes with Random Effects for Degradation Data," Journal of Multivariate Analysis, vol. 101, no. 2, pp. 340-351, 2010

26. G.A. Whitmore, "Estimating Degradation by A Wiener Diffusion Process Subject to Measurement Error," Lifetime data analysis, vol. 1, no. 3, pp. 307-319, 1995

27. A. Elwany, and N. Gebraeel, "Real-time estimation of mean remaining life using sensor-based degradation models," Journal of manufacturing science and engineering, vol. 131, no. 5, pp. 051005-1-9, 2009

28. X.S. Si, W. Wang, C.H. Hu, D. H Zhou, and M.G. Pecht, "Remaining Useful Life Estimation Based on a Nonlinear Diffusion Degradation Process," IEEE Transactions on Reliability, vol. 61, no. 1, pp. 50-67, 2012

29. X. Wang, N. Balakrishnan, and B. Guo, "Residual Life Estimation Based On a Generalized Wiener Degradation Process," Reliability Engineering \& System Safety, vol. 124, pp. 13-23, 2014

30. J.F. Zheng, X.S. Si, C.H. Hu, Z.X Zhang, and W. Jiang, "A Nonlinear Prognostic Model for Degrading Systems with ThreeSource Variability," IEEE Transactions on Reliability, vol. 65, no. 2, pp. 736-750, 2016

31. Z.S. Ye, Y Wang, K.L Tsui, and M. Pecht, "Degradation Data Analysis Using Wiener Processes with Measurement Errors," Transactions on Reliability, vol. 62, no. 4, pp. 772-780, 2013

32. C.Y. Peng, and S.T Tseng, "Mis-Specification Analysis of Linear Degradation Models," IEEE Transactions on Reliability, vol. 58, no. 3, pp. 444-55, 2009

33. X.S. Si, W. Wang, C.H. Hu, M. Chen, and D.H Zhou, "A Wiener-Process-Based Degradation Model with A Recursive Filter Algorithm for Remaining Useful Life Estimation,” Mechanical Systems and Signal Processing, vol. 35, no. 1, pp. 219-37, 2013

34. C.Y. Peng, and S.T Tseng, "Statistical Lifetime Inference with Skew-Wiener Linear Degradation Models," IEEE Transactions on Reliability, vol. 62, no. 2, pp. 338-50, 2013

35. Z.S. Ye, N. Chen, Y. Shen, "A New Class of Wiener Process Models for Degradation Analysis.," Reliability Engineering \& System Safety, vol. 139, pp. 58-67, 2015

36. H. Liao, and Z. Tian, "A Framework for Predicting the Remaining Useful Life of a Single Unit Under Time-Varying Operating Conditions," IIE Transactions, vol. 45, no. 9, pp. 964-80, 2013

37. T. Liu, Q. Sun, J. Feng, Z. Pan, and Q. Huangpeng, "Residual Life Estimation under Time-varying Conditions Based on a Wiener Process," Journal of Statistical Computation and Simulation, vol. 87, no. 2, pp. 211-226, 2017

38. E.A. Elsayed, "Reliability Engineering," Addison Wesley Longman, 1996, New York, NY

39. L. Bian, N. Gabraeel, and J.P. Kharoufeh, "Degradation Modeling for Real-Time Estimation of Residual Lifetimes in Dynamic Environments," IIE Transactions, vol. 47, no. 5, pp. 2471-86, 2015

40. S.J. Bae, and T. Yuan, S. Ning, W.A. Kuo, "A Bayesian Approach to Modeling Two-Phase Degradation Using Change-Point Regression," Reliability Engineering \& System Safety, vol. 134, pp. 66-74, 2015

41. M.A. Abdel-Hameed, “A Gamma Wear Process,” IEEE transactions on Reliability, vol. 24, no. 2, pp. 152-153, 1975

42. A. Grall, C. Bérenguer, L.A. Dieulle, "A Condition-Based Maintenance Policy for Stochastically Deteriorating Systems," 
Reliability Engineering \& System Safety, vol. 76, no. 2, pp. 167-80, 2002

43. J. Lawless, and M. Crowder, "Covariates and Random Effects in A Gamma Process Model with Application to Degradation and Failure," Lifetime Data Analysis, vol. 10, no. 3, pp. 213-27, 2004

44. J.M. Van Noortwijk, "A survey of the application of gamma processes in maintenance," Reliability Engineering \& System Safety, vol. 94 , no. 1, pp. 2-21, 2009

45. M.D. Pandey, and X.X. Yuan, J.M. Van Noortwijk, "The influence of temporal uncertainty of deterioration on life-cycle management of structures," Structure and Infrastructure Engineering, vol. 5, no. 2, pp. 145-156, 2009

46. Z.S. Ye, M. Xie, L.C. Tang, and N. Chen, "Semiparametric Estimation of Gamma Processes for Deteriorating Products," Technometrics, vol. 56, no. 4, pp. 504-13, 2014

47. D. Lu, M.D. Pandey, and W.C. Xie, "An Efficient Method for The Estimation of Parameters of Stochastic Gamma Process from Noisy Degradation Measurements.," Proceedings of the Institution of Mechanical Engineers, Part O: Journal of Risk and Reliability, vol. 227, no. 4, pp. 425-33, 2013

48. K. Le Son, M. Fouladirad, A. Barros, "Remaining Useful Lifetime Estimation and Noisy Gamma Deterioration Process," Reliability Engineering \& System Safety, vol. 149, pp. 76-87, 2016

49. G.A. Pulcini, "A Perturbed Gamma Process with Statistically Dependent Measurement Errors," Reliability Engineering \& System Safety, vol. 152, no. 4, pp. 152:296-306, 2016

50. V. Bagdonavicius, and M.S. Nikulin, "Estimation in Degradation Models with Explanatory Variables," Lifetime Data Analysis, vol. 7, no. 1, pp. 85-103, 2001

51. W. Peng, Y.F. Li, Y.J. Yang, J. Mi, and H.Z. Huang, "Leveraging Degradation Testing and Condition Monitoring for Field Reliability Analysis with Time-varying Operating Missions," IEEE Transactions on Reliability, vol. 64, no. 4, pp. 1367-82, 2015

52. B. Guo, and L. Tan, "Reliability Assessment of Gamma Deteriorating System Based on Bayesian Updating," 8th International Conference on Reliability, Maintainability and Safety, pp. 429-432, 2009

53. X. Wang, and D. Xu, "An Inverse Gaussian Process Model for Degradation Data," Technometrics, vol. 52, no. 2, pp. 188-97, 2010

54. H. Qin, S. Zhang, and W. Zhou, "Inverse Gaussian Process-Based Corrosion Growth Modeling and Its Application in The Reliability Analysis for Energy Pipelines," Frontiers of Structural and Civil Engineering, vol. 7, no. 3, pp. 276-87, 2013

55. Z.S. Ye, N. Chen, "The Inverse Gaussian Process as A Degradation Model,” Technometrics, vol. 56, no. 3, pp. 302-11, 2014

56. W. Peng, Y.F. Yang, H.Z. Huang, and M.J. Zuo, "Inverse Gaussian Process Models for Degradation Analysis: A Bayesian Perspective," Reliability Engineering \& System Safety, vol. 130, pp. 175-89, 2014

57. C.Y. Peng, "Inverse Gaussian Processes with Random Effects and Explanatory Variables for Degradation Data," Technometrics, vol. 57, no. 1, pp. 100-111, 2015

58. R. Moghaddass, and M.J. Zuo, "A Parameter Estimation Method for A Condition-Monitored Device Under Multi-State Deterioration," Reliability Engineering \& System Safety, vol. 106, pp. 94-103, 2012

59. R. Moghaddass, M.J. Zuo, and X. Zhao, "Modeling Multi-State Equipment Degradation with Non-Homogeneous ContinuousTime Hidden Semi-Markov Process," Diagnostics and Prognostics of Engineering Systems: Methods and Techniques, vol. 151, no. 8, pp. 599-612, 2012

60. A. Chen, and G.S. Wu, "Real-Time Health Prognosis and Dynamic Preventive Maintenance Policy for Equipment Under Aging Markovian Deterioration," International Journal of Production Research, vol. 45, no. 15, pp. 3351-79, 2007

61. C.T. Chen," Dynamic Preventive Maintenance Strategy for an Aging and Deteriorating Production System," Expert Systems with Applications, vol. 38, no. 5, pp. 6287-93, 2011

62. M. Compare, F. Martini, S. Mattafirri, F. Carlevaro, and E. Zio, "Semi-Markov model for the oxidation degradation mechanism in gas turbine nozzles," IEEE Transactions on Reliability, vol. 65, no. 2, pp. 574-81, 2016

63. O. Chryssaphinou, N. Limnios, and S. Malefaki, "Multi-State Reliability Systems Under Discrete Time Semi-Markovian Hypothesis," IEEE Transactions on reliability, vol. 60, no. 1, pp. 80-7, 2011

64. J.P. Kharoufeh, C.J. Solo, and M.Y. Ulukus, "Semi-Markov Models for Degradation-Based Reliability," IIE Transactions, vol. 42, no. 8, pp. 599-612, 2010

65. C. Bunks, D. McCarthy, and T. Al-Ani, "Condition-based maintenance of machines using hidden Markov models," Mechanical Systems and Signal Processing, vol. 14, no. 4, pp. 597-612, 2000

66. P. Baruah, R.B Chinnam, "HMMs for Diagnostics and Prognostics in Machining Processes," International Journal of Production Research, vol. 43, no. 6, pp. 1275-93, 2005

67. Z.J. Zhou, C.H. Hu, D.L. Xu, M.Y. Chen, and D.H. Zhou, "A Model for Real-Time Failure Prognosis Based On Hidden Markov Model and Belief Rule Base," European Journal of Operational Research, vol. 207, no. 1, pp. 269-83, 2010

68. M.E. Cholette, and D. Djurdjanovic," Degradation Modeling and Monitoring of Machines Using Operation-Specific Hidden Markov Models," IIE Transactions, vol. 46, no. 10, pp. 1107-23, 2014

69. D. Zhang, A.D. Bailey, and D. Djurdjanovic, "Bayesian Identification of Hidden Markov Models and Their Use for ConditionBased Monitoring," IEEE Transactions on Reliability, vol. 65, no. 3, pp. 1471-82, 2016

70. W. Wang, "A prognosis model for wear prediction based on oil-based monitoring," Journal of the Operational Research Society, vol. 58, no. 7, pp. 887-93, 2007

71. S.Z Yu, "Hidden Semi-Markov Models," Artificial Intelligence, vol. 178, no. 2, pp. 215-43, 2010

72. M. Dong, D. He, P. Banerjee, and J. Keller, "Equipment Health Diagnosis and Prognosis Using Hidden Semi-Markov Models," The International Journal of Advanced Manufacturing Technology, vol. 30, no. 7, pp. 738-49, 2006

73. M. Dong, and D. He, "Hidden Semi-Markov Model-Based Methodology for Multi-Sensor Equipment Health Diagnosis and Prognosis," European Journal of Operational Research, vol. 178, no. 3, pp. 858-78, 2007

74. Y. Peng, and M. A. Dong, "A Prognosis Method Using Age-Dependent Hidden Semi-Markov Model for Equipment Health 
Prediction," Mechanical Systems and Signal Processing, vol. 25, no. 1, pp. 237-52, 2011

75. Q, Liu, M. Dong, and Y. Peng, "A Novel Method for Online Health Prognosis of Equipment Based On Hidden Semi-Markov Model Using Sequential Monte Carlo Methods," Mechanical Systems and Signal Processing, vol. 32, pp. 331-48, 2012

76. Q. Liu, M. Dong, W. Lv, X. Geng, and Y, Li, “A Novel Method Using Adaptive Hidden Semi-Markov Model for Multi-Sensor Monitoring Equipment Health Prognosis, "Mechanical Systems and Signal Processing. vol. 64, pp. 217-32, 2015

77. N.Z. Gebraeel, and M.A. Lawley," A Neural Network Degradation Model for Computing and Updating Residual Life Distributions, "IEEE Transactions on Automation Science and Engineering, vol. 5, no. 1, pp. 154-63, 2008

78. A. Malhi, R. Yan, and R.X. Gao, "Prognosis of Defect Propagation Based On Recurrent Neural Networks," IEEE Transactions on Instrumentation and Measurement, vol. 60, no. 3, pp. 703-11, 2011

79. O. Fink, E. Zio, and U. Weidmann, "Predicting Component Reliability and Level of Degradation with Complex-Valued Neural Networks," Reliability Engineering \& System Safety, vol. 121, pp. 198-206, 2014

80. M.S. Kan, A.C. Tan, and J. Mathew, "A review on prognostic techniques for non-stationary and non-linear rotating systems," Mechanical Systems and Signal Processing, vol. 62, pp. 1-20, 2015

81. E. Zio, and F. Di Maio, "A Data-Driven Fuzzy Approach for Predicting the Remaining Useful Life in Dynamic Failure Scenarios of a Nuclear System,” Reliability Engineering \& System Safety, vol. 95, no. 1, pp. 49-57, 2010

82. F. Zhao, J. Chen, L. Guo, and X. Li, "Neuro-Fuzzy Based Condition Prediction of Bearing Health," Journal of Vibration and Control, vol. 15, no. 7, pp. 1079-91, 2009

83. A. Soualhi, H. Razik, G. Clerc, and D.D. Doan, "Prognosis of Bearing Failures Using Hidden Markov Models and The Adaptive Neuro-Fuzzy Inference System," IEEE Transactions on Industrial Electronics, vol. 61, no. 4, pp. 2864-74, 2014

84. M. Jouin, R. Gouriveau, D. Hissel, M.C. Péra, and N. Zerhouni, "Particle Filter-Based Prognostics: Review, Discussion and Perspectives, " Mechanical Systems and Signal Processing, vol. 72, pp. 2-31, 2016

85. J. Fan, K.C. Yung, and M. Pecht, "Physics-of-failure-based prognostics and health management for high-power white lightemitting diode lighting," IEEE Transactions on device and materials reliability, vol. 11, no. 3, pp. 407-16, 2011

86. P.C. Paris, and F. Erdogan, "A Critical Analysis of Crack Propagation Laws,” ASME, 1963.

87. R. Forman, V.E Kearney, RM. Engle, "Numerical Analysis of Crack Propagation in Cyclic Loaded Structures," Journal of basic Engineering, vol. 89, pp. 459-64, 1967

88. M. Pecht, and A. Dasgupta, "Physics-of-failure: an approach to reliable product development," Journal of the IES, vol. 38, no. 5, pp. 30-34, 1995

89. D.E. Adams, "Nonlinear damage models for diagnosis and prognosis in structural dynamic systems," International Society for Optics and Photonics, pp. 180-191, 2002

90. J. Gu, D. Barker, and M. Pecht, "Prognostics Implementation of Electronics Under Vibration Loading," Microelectronics reliability, vol. 47, no. 12, pp. 1849-56, 2007

91. C.S. Kulkarni, G. Biswas, J.R. Celaya, and K. Goebel, "Physics Based Degradation Models for Electrolytic Capacitor Prognostics Under Thermal Overstress Conditions," International Journal of Prognostics and Health Management, vol. 825, 2013

92. L. Yang, P.A. Agyakwa, and C.M. Johnson, "Physics-of-failure lifetime prediction models for wire bond interconnects in power electronic modules," IEEE Transactions on Device and Materials Reliability, vol. 13, no. 1, pp. 9-17, 2013

93. N. Li, Y. Lei, Z. Liu, J. Lin, "A Particle Filtering-Based Approach for Remaining Useful Life Predication of Rolling Element Bearings," Prognostics and Health Management (PHM), IEEE Conference, pp. 1-8, 2014

94. C.H. Oppenheimer, and K.A. Loparo, "Physically based diagnosis and prognosis of cracked rotor shafts," International Society for Optics and Photonics, pp. 122-132, 2002

95. W.K. Yu, and T.A. Harris, “A New Stress-Based Fatigue Life Model for Ball Bearings," Tribology transactions, vol. 44, no. 1, pp. $11-18,2001$

96. F. Zhao, Z. Tian, and Y. Zeng, "Uncertainty Quantification in Gear Remaining Useful Life Prediction Through an Integrated Prognostics Method," IEEE Transactions on Reliability, vol. 62, no. 1, pp. 146-59, 2013

97. V. Rathod, O.P. Yadav, A. Rathore, and R. Jain, "Probabilistic Modeling of Fatigue Damage Accumulation for Reliability Prediction," International Journal of Quality, Statistics, and Reliability, vol. 2011, 2011

98. J. Guang, G. Jin, Q. Liu, J. Zhou, and Z. Zhou, "Repofe: Reliability Physics of Failure Estimation Based On Stochastic Performance Degradation for The Momentum Wheel," Engineering Failure Analysis, vol. 22, pp. 50-63, 2012

99. D.C. Swanson, J.M. Spencer, and S.H. Arzoumanian, "Prognostic Modelling of Crack Growth in A Tensioned Steel Band. Mechanical Systems and Signal Processing, "vol. 14, no. 5, pp. 789-803, 2000

100. E. Zio, and G. Peloni, "Particle Filtering Prognostic Estimation of the Remaining Useful Life of Nonlinear Components," Reliability Engineering \& System Safety, vol. 96, no. 3, pp. 403-409, 2011

101. D. An, J.H. Choi, T.L. Schmitz, and N.H. Kim,” In Situ Monitoring and Prediction of Progressive Joint Wear Using Bayesian Statistics," Wear, vol. 270, no. 11, pp. 828-838, 2011

102. V. Crk," Reliability Assessment from Degradation Data," In Reliability and Maintainability Symposium, IEEE, pp. 155-161, 2000

103. P. Wang, and D.W. Coit, "Reliability Prediction Based On Degradation Modeling for Systems with Multiple Degradation Measures," In Reliability and Maintainability Annual Symposium-RAMS, pp. 302-307, 2004

104. J.K. Sari, "Multivariate Degradation Modeling and Its Application to Reliability Testing," PhD thesis, National University of Singapore, Singapore, 2007

105. Z. Pan, N. Balakrishnan, Q. Sun, and J. Zhou, "Bivariate Degradation Analysis of Products Based On Wiener Processes and Copulas," Journal of Statistical Computation and Simulation, vol. 83, no. 7, pp. 1316-29, 2013

106. W. Peng, Y.F. Li, Y.J. Yang, S.P. Zhu, and H.Z. Huang," Bivariate Analysis of Incomplete Degradation Observations Based On Inverse Gaussian Processes and Copulas," IEEE Transactions on Reliability, vol. 65, no. 2, pp. 624-39, 2016a

107. W. Peng, Y.F. Li, J. Mi, L. Yu, and H.Z. Huang," Reliability of Complex Systems Under Dynamic Conditions: A Bayesian 
Multivariate Degradation Perspective," Reliability Engineering \& System Safety, vol. 153, pp. 75-87, 2016b

108. R.P. Nicolai, and R. Dekker, "Optimal Maintenance of Multi-Component Systems: A Review," In Complex system maintenance handbook, Springer London, pp. 263-286, 2008

109. J. Li, D.W. Coit, E.A. Elsayed, "Reliability Modeling of a Series System with Correlated or Dependent Component Degradation Processes", International Conference on Quality, Reliability, Risk, Maintenance, and Safety Engineering (ICQR2MSE), pp. 388393, 2011

110. Z. Liang, and A.K. Parlikad, "A Condition-Based Maintenance Model for Assets with Accelerated Deterioration Due to Fault Propagation," IEEE Transactions on Reliability, vol. 64, no. 3, pp. 972-82, 2015

111. L. Bian, and N. Gebraeel, "Stochastic Modeling and Real-Time Prognostics for Multi-Component Systems with Degradation Rate Interactions," IIE Transactions, vol. 46, no. 5, pp. 470-82, 2014a

112. L. Bian, and N. Gebraeel, "Stochastic Framework for Partially Degradation Systems with Continuous Component DegradationRate-Interactions," Naval Research Logistics, vol. 61, no. 4, pp. 286-303, 2014b

113. S. Eryilmaz, "Modeling Dependence Between Two Multi-State Components Via Copulas," IEEE Transactions on Reliability, vol. 63 , no. 3, pp. 715-20, 2014

114. C.D. Dao, and M.J Zuo, "Selective Maintenance for Multistate Series Systems with S-Dependent Components," IEEE Transactions on Reliability, vol. 65, no. 2, pp. 525-39, 2016

115. G.A. Klutke, and Y. Yang, "The Availability of Inspected Systems Subject to Shocks and Graceful Degradation," IEEE Transactions on Reliability, vol. 51, no. 3, pp. 371-374, 2002

116. W. Li, and H. Pham, "An Inspection-Maintenance Model for Systems with Multiple Competing Processes," IEEE Transactions on Reliability, vol. 54, no. 2, pp. 318-27, 2005

117. Y. Wang, and H. Pham, "A Multi-Objective Optimization of Imperfect Preventive Maintenance Policy for Dependent Competing Risk Systems with Hidden Failure,” IEEE Transactions on Reliability, vol. 60, no. 4, pp. 770-81, 2011

118. Z. Wang, H.Z. Huang, Y. Li, N.C. Xiao, “An Approach to Reliability Assessment Under Degradation and Shock Process," IEEE Transactions on Reliability, vol. 60, no. 4, pp. 852-63, 2011

119. L. Jiang, Q. Feng, and D.W. Coit, "Reliability and Maintenance Modeling for Dependent Competing Failure Processes with Shifting Failure Thresholds," IEEE Transactions on Reliability, vol. 61, no. 4, pp. 932-48, 2012

120. X. Liu, J. Li, K.N. Al-Khalifa, A.S. Hamouda, D.W. Coit, and E.A. Elsayed, "Condition-Based Maintenance for Continuously Monitored Degrading Systems with Multiple Failure Modes," IIE Transactions, vol. 45, no. 4, pp. 422-35, 2013

121. K. Rafiee, Q. Feng, D.W. Coit, "Reliability Modeling for Dependent Competing Failure Processes with Changing Degradation Rate, “IIE transactions, vol. 46, no. 5, pp. 483-96, 2014

122. L. Jiang, Q. Feng, D.W. Coit, "Modeling Zoned Shock Effects On Stochastic Degradation in Dependent Failure Processes,” IIE Transactions, vol. 47, no. 5, pp. 460-70, 2015

123. Y. Wang, H. Pham, "Modeling The Dependent Competing Risks with Multiple Degradation Processes and Random Shock Using Time-Varying Copulas," IEEE Transactions on Reliability, vol. 61, no. 1, pp. 13-22, 2012

124. S. Song, D.W. Coit, Q. Feng, and H. Peng, "Reliability Analysis for Multi-Component Systems Subject to Multiple Dependent Competing Failure Processes," IEEE Transactions on Reliability, vol. 63, no. 1, pp. 331-45, 2014

125. N.C. Caballé, I.T. Castro, C.J. Pérez, and J.M. Lanza-Gutiérrez, "A Condition-Based Maintenance of a Dependent DegradationThreshold-Shock Model in A System with Multiple Degradation Processes," Reliability Engineering \& System Safety, vol. 134, pp. 98-109, 2015

126.M.J.Kim, V. Makis, and R. Jiang, "Parameter Estimation for Partially Observable Systems Subject to Random Failure," Applied Stochastic Models in Business and Industry, vol. 29, no. 3, pp. 279-94, 2013

\section{Author's Biography}

Ameneh Forouzandeh Shahraki is currently working towards a Ph.D. degree at the North Dakota state University. Her research interest is focused on reliability-based robust design optimization and for improving reliability, maintainability, and availability by using degradation-based data. She is a member of the Institute of Industrial and System Engineers (IISE) and ASQ Reliability Division.

Om Prakash Yadav is a Professor and Chair in the department of Industrial and Manufacturing Engineering Department at North Dakota State University, Fargo, North Dakota. He received his BS in Mechanical Engineering from MNIT, Jaipur (India) and MS in Industrial Engineering from NITIE, Mumbai (India). He completed his Ph.D. degree in Industrial Engineering from Wayne State University, Detroit, USA. He has over 30 years of teaching, research, industrial, and consulting experience. His research interests are focused on modelling complex system reliability, degradation behaviour, risk assessment, maintenance and spare parts inventory, robust design optimization, and manufacturing systems analysis. He was the winner of the 2015 William A.J. Golomski Best Paper Award. He is a member of IISE, ASQ, and SRE.

Haitao Liao is a Professor and Hefley Endowed Chair of Logistics and Entrepreneurship in the Industrial Engineering Department at the University of Arkansas, Fayetteville, Arkansas, USA. He received his Ph.D. degree in 2004 from the 
Department of Industrial and Systems Engineering at Rutgers University. His research interests are focused on modeling of accelerated testing, probabilistic risk assessment, maintenance models and optimization, spare part inventory control, and prognostics. He was a recipient of the National Science Foundation CAREER Award in 2010 and the winner of the 2010 \& 2013 William A. J. Golomski Best Paper Award. 